\title{
A method for inspecting near-right-angle V-groove surfaces based on dual-probe wavelength scanning interferometry
}

\author{
Tao Zhang ${ }^{1} \cdot$ Feng Gao ${ }^{1} \cdot$ Haydn Martin ${ }^{1} \cdot$ Xiangqian Jiang $^{1}$ \\ Received: 22 November 2017 / Accepted: 6 June 2018 / Published online: 12 July 2018 \\ (C) The Author(s) 2018
}

\begin{abstract}
High-angled structured surfaces such as micropyramidal arrays, V-grooves and lenslet arrays are widely used in industries. However, there is currently no effective way to inspect these microstructures, resulting in very high scrap rates. This paper presents a proof-of-principle demonstration of an optical system capable of measuring V-groove structures in a single measurement acquisition. The dual-probe wavelength scanning interferometry (DPWSI) system comprises dual probes, with orthogonal measurement planes. The calibration of the DPSWI system is the key to registering the relative locations of the dual measurement planes and allowing the surface topography to be correctly reconstructed. In order to achieve this, a custom calibration artefact was manufactured comprising focused ion beam etched features on two faces of a precision cube. The procedures for the characterisation of the artefact to generate reference topography, and the subsequent calibration of the DPWSI are described in full. A measurement example from a metallised saw tooth sample featuring near-right-angles grooves having a peak-to-valley height of $32 \mu \mathrm{m}$ and nominal pitch of $25 \mu \mathrm{m}$ is presented and compared with a result obtained using stylus profilometry. DPWSI is shown to obtain an areal dataset in a single acquisition and is able to better resolve peak/valley points compared with the stylus, which is limited by a $2-\mu \mathrm{m}$ tip radius. Some lateral scale error is apparent in the final DPWSI results and a discussion of this in terms of the limitations surrounding the current calibration artefact is presented.
\end{abstract}

Keywords Surface metrology $\cdot$ Interferometry $\cdot$ Structured surfaces $\cdot$ Calibration

\section{Introduction}

An important and developing need in surface metrology is to be able to measure structured and freeform surfaces [1]. Micro-fabricated structured surfaces with multiple highangle facets are widely applied in many application areas [2-4]. For example, brightness enhancement film has a prismatic structure and is frequently used in liquid crystal displays to enable power saving and thermal management. Optical gratings comprising periodic structures find widespread applications in optical instruments such as spectrometers, lasers, wavelength division multiplexing devices, etc. [5-9]. However, in general, the manufacturing processes to create such surfaces are heavily reliant on the experience of fabrication workers adopting an expensive trial-and-error approach,

Feng Gao

F.Gao@hud.ac.uk

1 University of Huddersfield, Huddersfield HD1 3DH, UK resulting in the reporting of high scrap rates of up to $50-70 \%$ [10]. In this context, it is clear that overcoming the challenges of providing the effective measurement of these surfaces types will have a meaningful impact in terms of improving processes and reducing product costs.

Currently, the contact stylus profilometer and optical instruments are predominantly applied to inspect these types of structures. Stylus profilometery features the required vertical resolution but is potentially destructive, especially given many structured surfaces may be produced in polymers e.g. prismatic films. Additionally, areal measurement is relatively time-consuming with a stylus because the sample must be scanned line-by-line. Finally, the size of the stylus tip can induce large errors when measuring microstructures with high aspect ratios due to mechanical filtering [11]. Optical measurement techniques, such as confocal microscopy, focus variation and interferometry, are commonly limited by the numerical aperture (NA) of the objective lens which sets a limit on the maximum measurable slope angle [12]. Light reflected from facets that exceeds the acceptance angle associated with the objective lens is not collected and cannot be analyzed, 
leading to missing data or reduced precision. Additionally, multiple reflections can occur in V-grooves and other similar structures which results in severe measurement errors in optical instruments, particularly in coherence scanning interferometers (CSI) [9]. Other types of imaging techniques are also very restricted in this area, for instance scanning electron microscopy (SEM) provides high lateral resolution and depth of focus, but cannot acquire the height information directly and additionally; sample preparation can be non-trivial [13].

The measurement system reported in this paper aims to solve one commonly encountered high-angled structured surface feature, namely the V-groove. The method relies on wavelength scanning interferometry (WSI) [14-16] which can achieve measurement with axial resolutions approaching the nanometre [17] without the requirement for the mechanical scanning of either the sample or optics, unlike comparable techniques such as CSI. This lack of mechanical scanning opens up the possibility of using a dual-probe optics system to provide simultaneous measurement with two adjacent fields of view. This technique, which we term dual-probe wavelength scanning interferometry (DPWSI) is described in the following section.

\section{Methodology}

The DPWSI system, as illustrated in Fig. 1, comprises three modules: light source, interferometer and console. The light source consists of a tungsten-halogen filament source which is coupled to an acousto-optical tunable filter (AOTF). The AOTF filters the white light from the tungsten-halogen bulb into a narrowband wavelength (linewidth of $\sim 2 \mathrm{~nm}$, selectable across a range between 590.98 and $683.42 \mathrm{~nm}$ ) which is then coupled into a multimode fiber before subsequently illuminating the interferometer module. The interferometer module is composed of two cameras and two identical $\times 4$ magnification Michelson interference objectives (OBJ1, OBJ2). The two objectives are of a long working distance design $(30 \mathrm{~mm})$ and are placed such that their optical axes are orthogonal and their focal planes intersect. A beamsplitter (BS3) is used to split the collimated light from the multimode fiber and illuminate both objectives. The console acts as the controller and interface for the system as a whole. During the measurement process, the wavelength scanning of the illumination light is controlled and synchronised by a software running on the PC via the AOTF driver. A total of 256 spectral interferograms, each at a discrete wavelength, are captured from each of the Michelson objectives by their corresponding cameras. The two interferograms sets are then analyzed to acquire the surface topography under each respective probe using a suitable fringe analysis algorithm [15-18].

The measurable axial range for a WSI system is determined by the lesser of either (a) the coherence length of the illuminating light or (b) the depth of field (DoF) of the Michelson objective. In the apparatus described, the limit is found to be due to the objective DoF and is approximately $110 \mu \mathrm{m}$. The lateral measurement range is determined by the field of view (FOV) of the optical system. The Rayleigh criterion defines a lateral resolution for the system of $4.1 \mu \mathrm{m}$ as defined by the objective numerical aperture $(\mathrm{NA}=0.1)$ and the longest illumination wavelength.

For each objective, interference is generated between the light reflected from a reference mirror (REF1, REF2) and the light reflected from the measurand. In WSI, a virtual reference plane exists in the measurement path at the point at which the interferometer is balanced. Results obtained from each interferometer yield the optical path difference (OPD) from the measurand surface to the virtual reference planes (VREF1, VREF2). Because there is no mechanical movement during the measurement process, all the optics are stationary and thus the virtual reference planes VREF1 and VREF2 are nominally static. Therefore, if the relative location of the VREF1 and VREF2 can be established through calibration, then the topography of the sample as measured by the combination of both interferometers can be recovered.

The positional relationship between the two probes is built through the spatial coordinate calibration (Fig. 2). Because there is no overlapping measurement area between the two probes, traditional calibration methods are not applicable, thus a custom calibration artefact has been designed and manufactured. Figure 3 shows the calibration artefact which comprises a precision cube with two adjoining faces (labelled plane 1/2 in Fig. 3) certified to be perpendicular within $2 \mathrm{~s}$ of arc (the actual deviation measured by autocollimator is 1.2 arcsecs). The two faces of the cube have flatness better than $50 \mathrm{~nm}$ and $S_{a}$ roughness of less than $30 \mathrm{~nm}$. Upon each face, an array of $50 \mu \mathrm{m}$ square wells, having a depth of approximately $200 \mathrm{~nm}$ are etched using a focused ion beam (FIB). To our knowledge from both the literature and experiment, no existing instruments are able to directly measure the topography of the whole artefact with sufficient resolution to detect the features from a single orientation. As such, the acquisition of the reference topography was accomplished by combining the results obtained from CSI (Taylor Hobson CCI 3000) and SEM (FEI Quanta 200 3D SEM-FIB) instruments. CSI was adopted to measure the areas containing the etched features on each cube face separately. SEM was then applied to acquire relative locations between the features $\left(f_{1}, f_{2}\right)$ on the two faces, namely the distances $d_{1}, d_{2}$ and $d_{3}$ as shown in Fig. 3. SEM has a large enough depth of field to image all the features on both faces, while also providing the ability to determine distances with submicron resolution. Since the dihedral angle between the two faces is already known, the reference topography can be reconstructed by binding the datasets together based on the coordinate system shown in Fig. 3c by satisfying Eq. (1). 


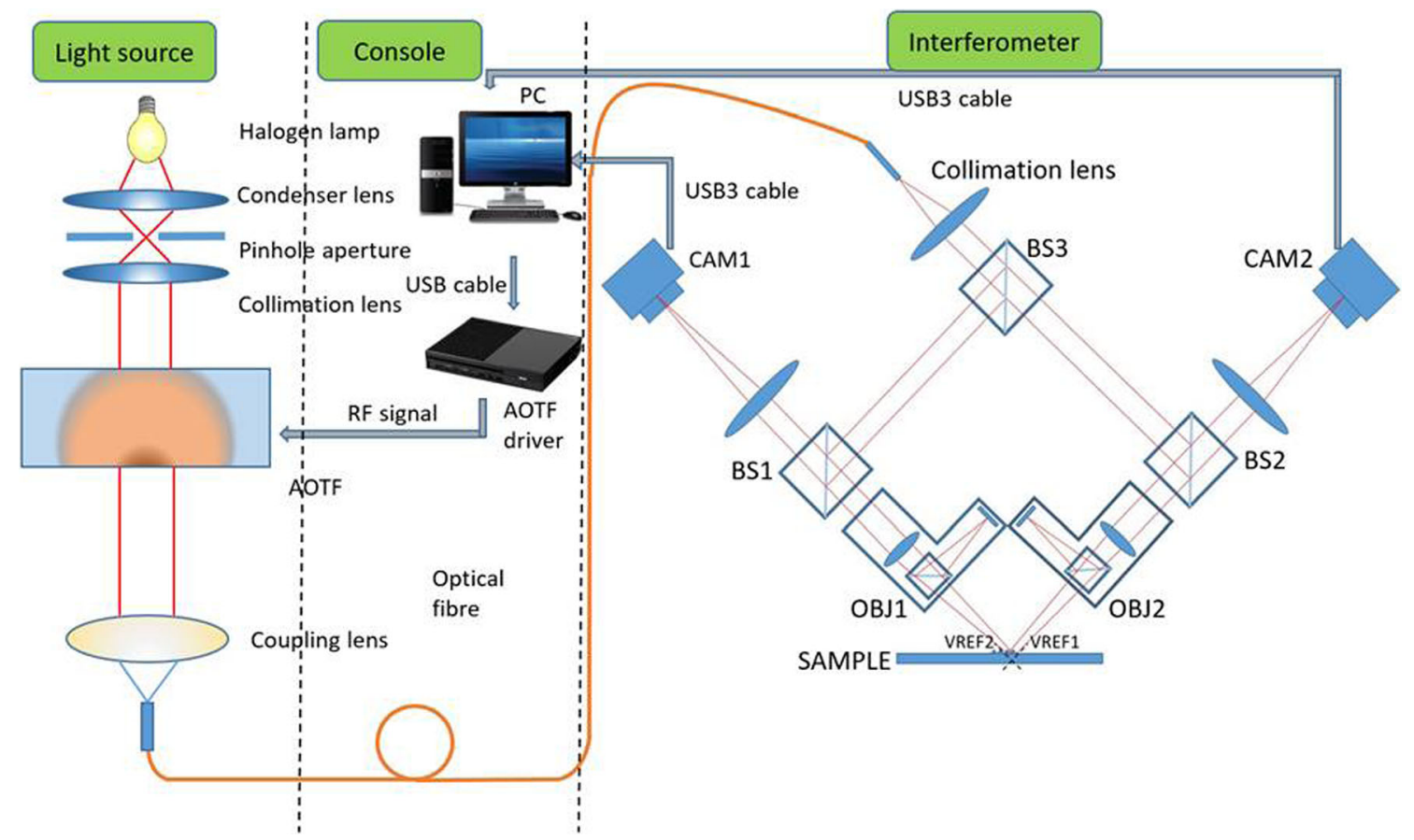

Fig. 1 Block diagram of the DPWSI system showing from left to right: the light source, console and dual-probe interferometer

$$
\left\{\begin{array}{l}
\text { distance }\left(f_{1}, p_{2}\right)=d_{1} \\
\text { distance }\left(f_{2}, p_{1}\right)=d_{2} \\
\text { distance }\left(f_{1}, f_{2}\right)=d_{3}
\end{array}\right.
$$

Here, $P_{n}$ signifies the fitted plane of face $n=1$ or $n=2, f_{m}$ refers to the specific feature on the artefact. This can be accomplished by maintaining one dataset, while rotating and translating the other dataset to satisfy these restrictions using only rigid transformations.

The features in the measurement results are extracted by an image segmentation algorithm such as Sobel operator and watershed transformation $[19,20]$. By matching the features correspondingly to the reference result, the relationship between the coordinate systems of the two probes and the coordinate system of the reference topography can be determined. The relative orientation of the coordinate systems of the two probes can then be calculated. If $P_{n}$ and $P_{n}^{\prime}$ are the quaternions of the feature points such as corners or centers of the features in the reference topography, $Q_{n}$ and $Q_{n}^{\prime}$ represent the quaternions of the corresponding feature points in the measurement results by the two probes. The following equations must be satisfied:

$$
\begin{aligned}
& \left(\begin{array}{cc}
R_{1} & t_{1} \\
0 & 1
\end{array}\right) Q_{n}^{\prime}=P_{n}^{\prime} \\
& \left(\begin{array}{cc}
R_{2} & t_{2} \\
0 & 1
\end{array}\right) Q_{n}=P_{n}
\end{aligned}
$$

$$
\begin{aligned}
R_{n}= & \left(\begin{array}{cccc}
1 & 0 & 0 & 0 \\
0 & \cos \alpha_{n} & \sin \alpha_{n} & 0 \\
0 & -\sin \alpha_{n} & \cos \alpha_{n} & 0 \\
0 & 0 & 0 & 1
\end{array}\right) \\
& *\left(\begin{array}{cccc}
\cos \beta_{n} & 0 & -\sin \beta_{n} & 0 \\
0 & 1 & 0 & 0 \\
\sin \beta_{n} & 0 & \cos \beta_{n} & 0 \\
0 & 0 & 0 & 1
\end{array}\right) \\
& *\left(\begin{array}{cccc}
\cos \gamma_{n} & \sin \gamma_{n} & 0 & 0 \\
-\sin \gamma_{n} & \cos \gamma_{n} & 0 & 0 \\
0 & 0 & 1 & 0 \\
0 & 0 & 0 & 1
\end{array}\right) \\
t_{n}= & \left(\begin{array}{lll}
t_{n x} t_{n y} & t_{n z}
\end{array}\right)
\end{aligned}
$$

where $R_{1}, R_{2}, t_{1}$ and $t_{2}$ represent the rotation matrices and translation matrices from the coordinate systems of the two probes to the reference topography respectively. These matrices are functions of $\alpha_{n}, \beta_{n}$ and $\gamma_{n}$ which are the respective rotation angles, and $t_{n x}, t_{n y}$ and $t_{n z}$ which are the respective translation components, along the $x, y$ and $z$ axes. As such, there are 12 unknown independent variables in total. Theoretically, if there are a sufficient number of feature point pairs, it is possible to precisely determine the matrices. However, since there are inevitable errors when extracting the feature point pairs in a practical measurement, a 3D registration algorithm based on iterative closest point (ICP) is utilised to increase the matching accuracy 


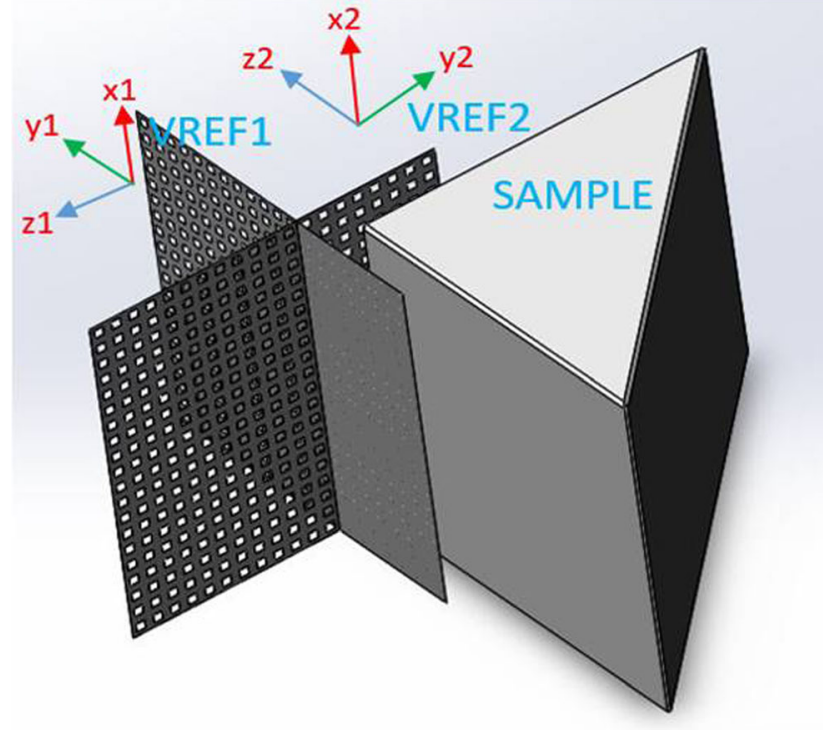

Fig. 2 An illustration of the coordinate systems and virtual reference planes of the two probes in the DPWSI apparatus

[21]. The datasets obtained by the two probes are bound together to acquire the whole topography of the structured surface as follows:

$$
\begin{aligned}
& \left(\begin{array}{c}
\left(\begin{array}{cc}
R_{1} & t_{1} \\
0 & 1
\end{array}\right) X \\
\left(\begin{array}{cc}
R_{2} & t_{2} \\
0 & 1
\end{array}\right) Y
\end{array}\right) \rightarrow\left(\begin{array}{c}
\left(\begin{array}{cc}
R_{1} & t_{1} \\
0 & 1
\end{array}\right)^{-1}\left(\begin{array}{cc}
R_{1} & t_{1} \\
0 & 1
\end{array}\right) X \\
\left(\begin{array}{cc}
R_{1} & t_{1} \\
0 & 1
\end{array}\right)^{-1}\left(\begin{array}{cc}
R_{2} & t_{2} \\
0 & 1
\end{array}\right) Y
\end{array}\right) \\
& =\left(\left(\begin{array}{cc}
R_{1} & t_{1} \\
0 & 1
\end{array}\right)^{-1}\left(\begin{array}{cc}
R_{2} & t_{2} \\
0 & 1
\end{array}\right) Y\right)
\end{aligned}
$$

where $X$ and $Y$ represent the datasets acquired by the two probes respectively. To minimise the computation, the result can be rotated and translated as a whole in the same coordinate system as shown in Eq. (6) with no change to the result.

\section{Experimental results and discussion}

The two probes of the DPWSI were first individually calibrated using a set of standard step-height specimens, after which the calibration artefact was measured. The stitched reference topography is shown in Fig. 4a, which was then used to calibrate the DPWSI system yielding the result shown in Fig. $4 \mathrm{~b}$.
Fig. 3 SEM images taken of the fabricated cubic calibration artefact showing key features $\left(f_{1}\right.$, $\left.f_{2}\right)$ and distances $\left(d_{1}, d_{2}, d_{3}\right)$ used in the calibration procedure. Note (a) and (b) are orientated such that the cube edge is shown at the bottom of the image in both cases

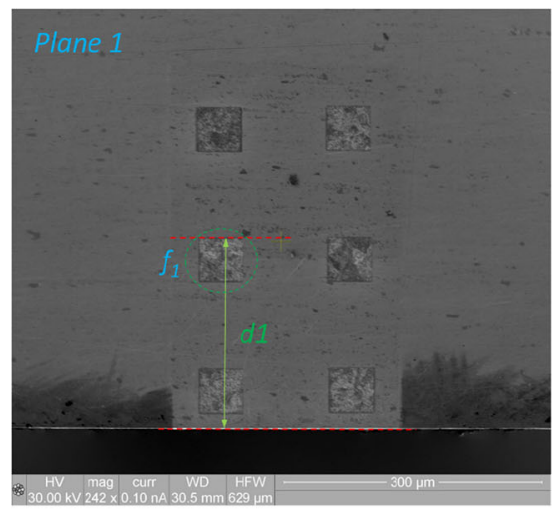

(a)

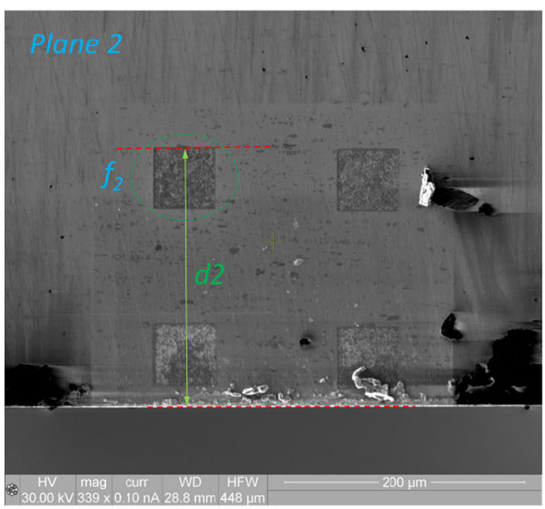

(b)

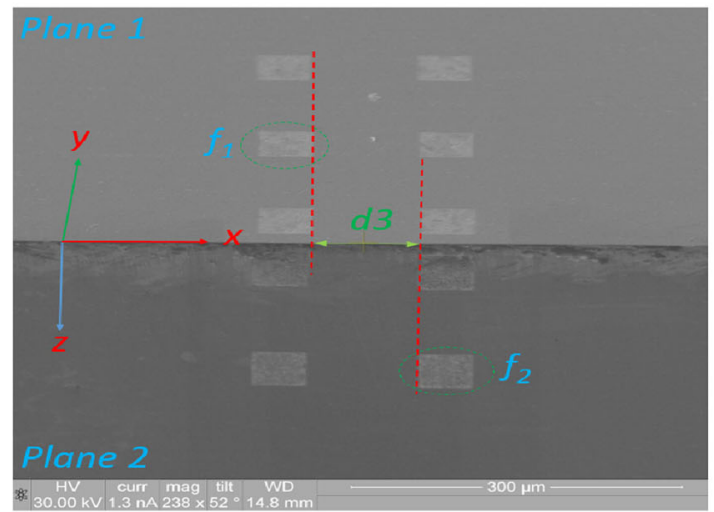

(c) 
Fig. 4 a The reference topography of the calibration artefact acquired using the combined CSI and SEM measurements described in the preceding section. $\mathbf{b}$ The calibration artefact measured by the DPWSI system. Both plotted with CloudCompare

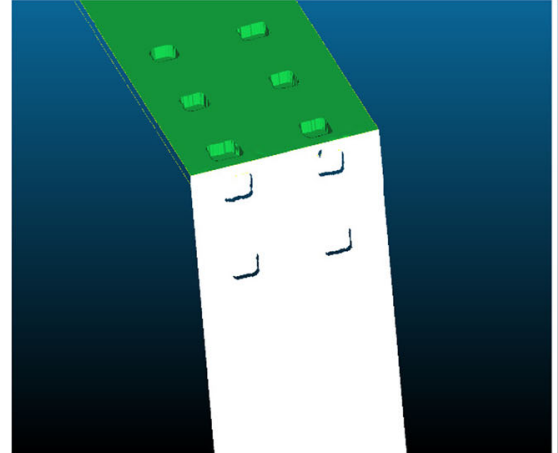

(a)

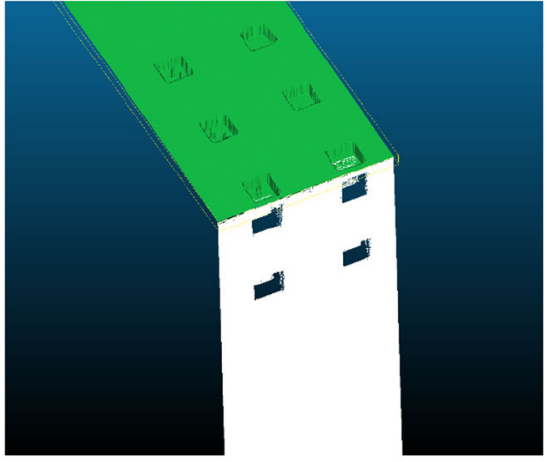

(b)
To verify the presented approach to V-groove measurement, a sawtooth metallised prismatic film manufactured by Microsharp Co. Ltd. was measured using the described DPWSI system. Figure 5 shows the individual data obtained from each probe and the combined result. The height of the measured step features is $\sim 32 \mu \mathrm{m}$ and the nominal pitch is $25 \mu \mathrm{m}$. It is apparent that in high curvature areas (peaks tops/ valley bottoms), some measurement data loss is experienced, which is due to the previously mentioned NA-dependent acceptance angle limitation for the optics [12]. Nonetheless, it is clear that a large proportion of the artefact has been successfully measured in a single acquisition, with the instrument in a single orientation.

Fig. 6a compares a profile taken from the DPWSI measurement result with one obtained across a similar region with a stylus profilometer (Taylor Hobson PGI Form Talysurf Series 2) using a $2-\mu \mathrm{m}$ tip radius stylus. The stylus profile was optimised using a deconvolution filter which provides some limited improvement in the obvious low-pass mechanical filtering exhibited by the tip radius in the peaks/valleys regions of the profile. For the DPWSI profile, the small amount of missing data apparent in Fig. 5 is also identifiable at the peaks/valleys of the profile, we have chosen not to interpolate this data; however, these regions where confirmed as being physically sharp using SEM imaging. The two profiles were registered using an ICP method to enable a stable comparison.

These results demonstrate that DPWSI can successfully recover a $\mathrm{V}$-groove profile in a single acquisition from a single measurement orientation, representing what we believe is a first for an optical measurement system. Careful evaluation
Fig. 5 Measurement data obtained from a metallised prismatic film: a probe 1 dataset, b probe 2 dataset, $\mathbf{c}$ combined dataset from probes 1 and 2 showing both aspects of the grooves in the sample

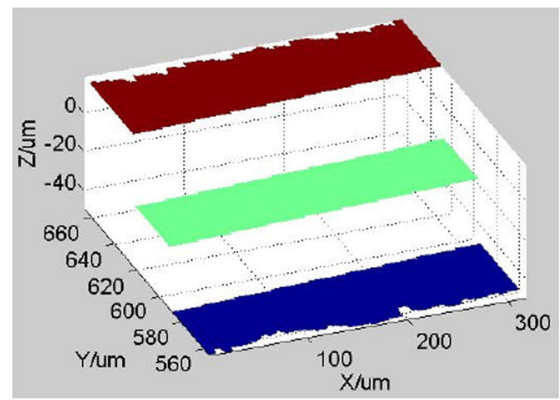

(a)

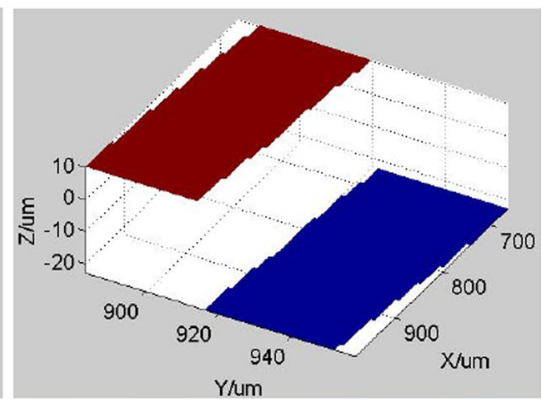

(b)

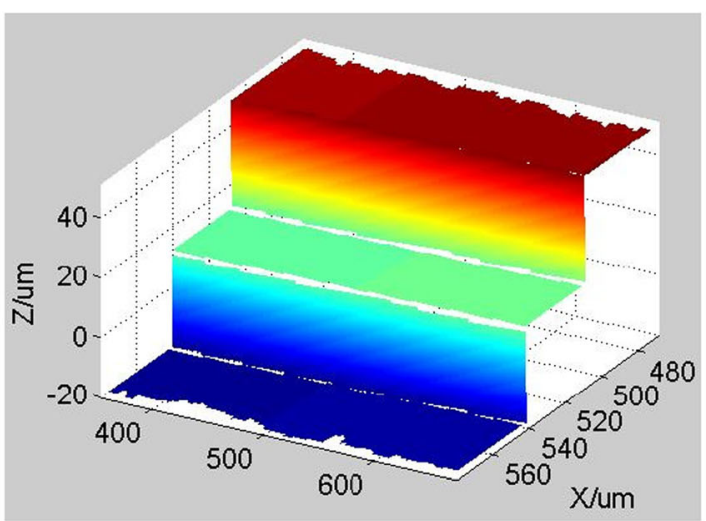

(c) 
Fig. 6 a The comparison of the measured profiles of the metallised prismatic film by the DPWSI (blue) and stylus profilometry (red). b Plot of the residual error between the DPWSI and stylus profiles obtained from the ICP algorithm used to register the two datasets
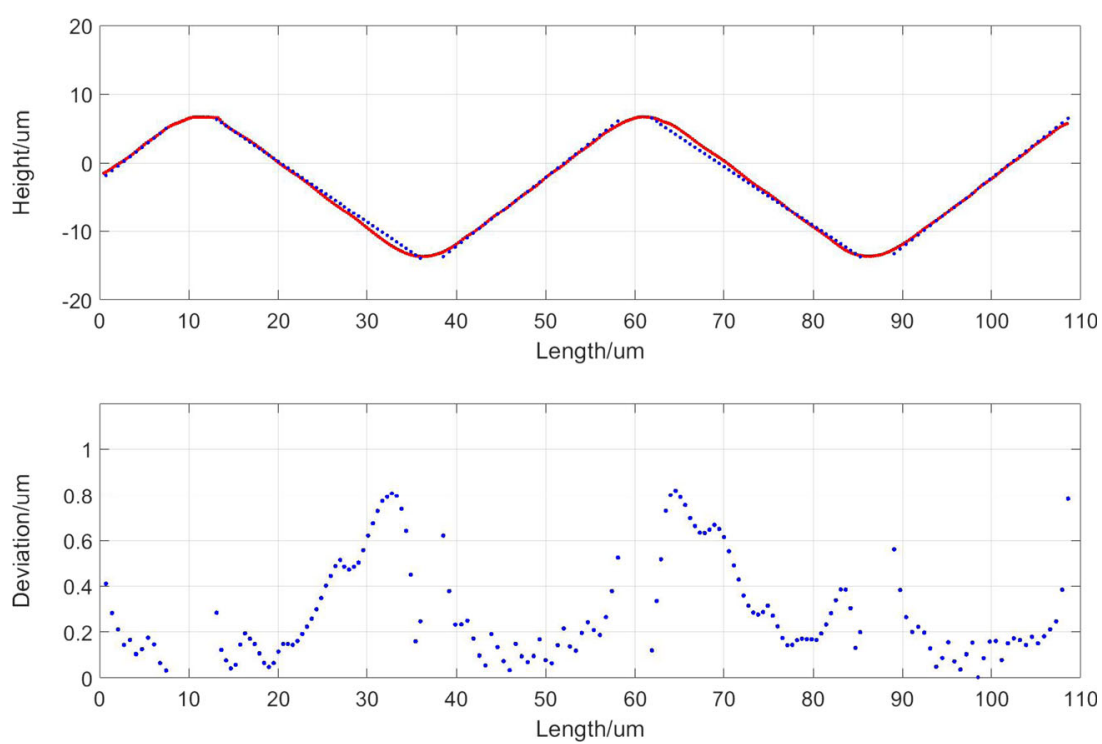

of Fig. 6a shows that there is some lateral scale error associated with the result from the DPWSI as compared with the profile obtained using stylus profilometry. Looking at the middle of the three obtained ridges, the left hand facet (measured by probe 1) exhibits a shortened scale (amplification coefficient $\sim 0.96$ ). Conversely, the scale for the right hand facet (measured by probe 2) is long (amplification coefficient 1.04 ) in relation to the stylus result. Figure $6 \mathrm{~b}$ shows the resulting residual error as obtained from the ICP algorithm along the profile. The error is seen to peak at approximately $820 \mathrm{~nm}$ but remains substantially less than this across most of the profile (the average value is $292.1 \mathrm{~nm}$ ). The residual error is contributed predominantly by the scale error previously discussed; however, there is an additional contribution from the mismatched sampling intervals of the two datasets. These scale errors are due to a systematic error introduced during the calibration process. In the calibration process, there are two primary error sources resulting from the image processing. Segmentation error resulting from inaccurate edge extraction is in part due to the imperfections in the fabricated features on the calibration artefact. Residual registration error derives not only from limitations in the $3 \mathrm{D}$ registration algorithm used, but also from mismatches between the obtained reference dataset and the measurement dataset. Considering all of the error sources, the residual error is quite reasonable since it is far less than the lateral resolution of the probes which is about $4 \mu \mathrm{m}$.

\section{Conclusion}

This paper demonstrates the principle and operation of an optical system capable of acute-angled V-groove measurement based upon a wavelength scanning interferometer coupled with a probe that provides two orthogonally located fields of view. A calibration procedure involving a custom designed calibration artefact was used to provide registration of the two fields. Experimental results demonstrate the measurement of a sawtooth profile from metallised film featuring a near-right-angled V-groove structure. For a given probe, a range of $\mathrm{V}$-groove angles are measurable, limited by the acceptance angle of the objective lenses employed. This range can be expanded further by designing equivalent probe heads with varying angular separation. Further work will examine in more detail the errors associated with the calibration procedure and aim to minimise these through a combination of improved artefact fabrication, improved reference topography construction, feature extraction and registration algorithm optimisation.

Funding information The study was funded by the UK's Engineering and Physical Sciences Research Council (EPSRC) of the Center for Innovative Manufacturing in Advanced Metrology (EP/I033424/1) and the EPSRC Future Advanced Metrology Hub (EP/P006930/1).

Open Access This article is distributed under the terms of the Creative Commons Attribution 4.0 International License (http:// creativecommons.org/licenses/by/4.0/), which permits unrestricted use, distribution, and reproduction in any medium, provided you give appropriate credit to the original author(s) and the source, provide a link to the Creative Commons license, and indicate if changes were made.

Publisher's Note Springer Nature remains neutral with regard to jurisdictional claims in published maps and institutional affiliations.

\section{References}

1. Jiang X, Whitehouse DJ (2012) Technological shifts in surface metrology. CIRP Ann Manuf Technol 61(2):815-836 
2. Zou H, Ding Y, Zhang J, Cai A, Zhang X, Zhou Y (2017) Precise geometric error model based on Z-axis motion platform of micro vgroove machine tools. Int J Adv Manuf Technol:1-6

3. Zhang X, Jiang L, Zeng Z, Fang F, Liu X (2015) High angular accuracy manufacture method of micro $\mathrm{v}$-grooves based on tool alignment by on-machine measurement. Opt Express 23(21): 27819-27828

4. Lin T, Lin M, Chao C (2017) A novel and rapid fabrication method for a high fill factor hexagonal microlens array using thermal reflow and repeating spin coating. Int J Adv Manuf Technol 92(1-8): 3329-3336

5. Evans CJ, Bryan JB (1999) "Structured", "textured" or “engineered" surfaces. CIRP Ann Manuf Technol 48(2):541-556

6. Purcell D, Suratkar A, Davies A, Farahi F, Ottevaere H, Thienpont $\mathrm{H}$ (2010) Interferometric technique for faceted microstructure metrology using an index matching liquid. Appl Opt 49(4):732-738

7. Li D, Cheung CF, Ren M, Zhou L, Zhao X (2014) Autostereoscopybased three-dimensional on-machine measuring system for microstructured surfaces. Opt Express 22(21):25635-25650

8. Li D, Cheung CF, Ren M, Whitehouse D, Zhao X (2015) Disparity pattern-based autostereoscopic 3D metrology system for in situ measurement of microstructured surfaces. Opt Lett 40(22):52715274

9. Gao F, Coupland J, Petzing J (2006) V-groove measurements using white light interferometry, in Photon06, Manchester, pp. 4-7

10. Singleton L, Leach R, Lewis A, Cui Z (2002) Report on the analysis of the MEMSTAND survey on Standardisation of MicroSystems Technology, MEMSTAND Project IST-2001-37682

11. Ju B, Chen Y, Zhang W, Fang F (2012) Rapid measurement of a high step microstructure with $90^{\circ}$ steep sidewall. Rev Sci Inst 83(1): 013706
12. Malacara D (2007) Optical shop testing Wiley

13. Tafti AP, Kirkpatrick AB, Alavi Z, Owen HA, Yu Z (2015) Recent advances in 3D SEM surface reconstruction. Micron 78:54-66

14. Jiang X, Wang K, Gao F, Muhamedsalih H (2010) Fast surface measurement using wavelength scanning interferometry with compensation of environmental noise. Appl Opt 49(15):2903-2909

15. Muhamedsalih H, Gao F, Jiang X (2012) Comparison study of algorithms and accuracy in the wavelength scanning interferometry. Appl Opt 51(36):8854-8862

16. Takeda M, Yamamoto H (1994) Fourier-transform speckle profilometry: three-dimensional shape measurements of diffuse objects with large heights steps and/or spatially isolated surfaces. Appl Opt 33(34):7829-7837

17. Moschetti G, Forbes A, Leach RK, Jiang X, O’Connor D (2016) Phase and fringe order determination in wavelength scanning interferometry. Opt exp 24:8997-9012

18. Zhang T, Gao F, Muhamedsalih H, Lou S, Martin H, Jiang X (2018) Improvement of the fringe analysis algorithm for wavelength scanning interferometry based on filter parameter optimization. Appl Opt 57:2227-2234

19. MacAulay GD, Senin N, Giusca CL, Leach RK (2016) Study of manufacturing and measurement reproducibility on a laser textured structured surface. Meas 94:942-948

20. MacAulay GD, Senin N, Giusca CL, Leach RK (2014) Comparison of segmentation techniques to determine the geometric parameters of structured surfaces. Surf Topog: Metro Prop 2(4):044004

21. Fitzgibbon AW (2003) Robust registration of 2D and 3D point sets. Imag and Vis Comp 21(13-14):1145-1153 